\title{
Considerable variability in the clinical and ciliary features of primary ciliary dyskinesia in patients with DNAH5 mutations
}

\author{
Zhuoyao Guo \\ Children's Hospital of Fudan University \\ Weicheng Chen \\ Children's Hospital of Fudan University \\ Mengru Li \\ Children's Hospital of Fudan University \\ Libo Wang \\ Children's Hospital of Fudan University \\ Liling Qian ( $\square$ llqian@126.com) \\ Children's Hospital of Fudan University
}

\section{Research Article}

Keywords: primary ciliary dyskinesia, DNAH5 gene, phenotypic heterogeneity, ciliary beat pattern

Posted Date: January 31st, 2022

DOI: https://doi.org/10.21203/rs.3.rs-1283519/v1

License: @ (i) This work is licensed under a Creative Commons Attribution 4.0 International License. Read Full License 


\section{Abstract}

Background: Primary ciliary dyskinesia (PCD) is a clinically and genetically heterogeneous disorder, but the relationship between genotype and phenotype is poorly established. We aimed to characterize the detailed clinical characteristics, ciliary phenotype and mutational spectrum of PCD patients with DNAH5 mutations.

Methods: Whole exome sequencing followed by targeted copy number variation (CNV) analysis was used to screen 114 Chinese patients with highly suspected PCD. In cases with DNAH5 mutation, detailed demographic and clinical data, as well as ciliary beat pattern and ciliary ultrastructure, were comprehensively reviewed.

Results: Thirty patients (median, 15.3 years; range, 0.6 to 43 years), who carried DNAH5 mutation, were identified. We characterized 38 novel and 7 known DNAH5 mutations (10 nonsense, 5 frameshift, 10 splicing, 16 missense mutations, 2 in-frame deletions and 2 large deletions). Despite bearing the same genotype, patients presented a highly variable phenotypic picture, including milder respiratory symptom, normal nasal nitric oxide levels (2/21, 9.5\%) and "nontypical" ciliary beat pattern (4/21, 19.0\%).

Conclusions: DNAH5-associated PCD disease demonstrates dramatic phenotypic heterogeneity, contributing to the challenges of diagnosis. A composite work-up and cautious interpretation of the results are necessary during the PCD diagnosis process.

\section{Introduction}

Primary ciliary dyskinesia (PCD) is a rare, heterogeneous ciliopathy resulting in mucociliary clearance failure. Patients typically present with newborn respiratory distress, daily wet cough, chronic nasal congestion, and laterality defects. PCD diagnosis can be achieved by following the diagnostic algorithms that include nasal nitric oxide (nNO) measurements, high-speed video microscopy analysis (HSVA), immunofluorescence (IF), transmission electron microscopy (TEM), and molecular testing ${ }^{[1]}$. Currently, around 50 genes are known to be associated with PCD ${ }^{[2]}$.

The relationship between genotype and phenotype in PCD has been considered a matter of critical importance ${ }^{[3]}$, as better understanding of genotypephenotype correlations helps to promote more accurate prognostic assessments. It has been reported that patients with a $C C D C 39$ or $C C D C 40$ mutation have lower body mass indices and worse lung function that declines more rapidly compared with those with $D N A H 5$ mutations ${ }^{[4]}$. Conversely, patients with $R S P H 1$ and $D N A H 9$ mutations appear to have a milder respiratory phenotype $\mathrm{e}^{[5,6]}$.

The ciliary ultrastructural and beat patterns correlate with specific genotypes. Specifically, mutations in CCDC39 or CCDC40 cause axonemal disorganization and loss of inner dynein arms, conferring extremely stiff cilia with reduced beat amplitude ${ }^{[7]}$. Mutations in genes that encode cytoplasmic preassembly factors, such as $D N A A F 3$, result in complete cilia immotility, with combined outer and inner dynein arm defects ${ }^{[8]}$. The consistency across ciliary beating anomalies and specific genotypes makes HSVA a promising approach to assess the pathogenicity of VUS variants. However, extensive variability in the clinical and ciliary phenotype within a single mutation cohort has also been reported. In a study of CCDC103 p. His 154 Pro patients by Shoemark et al., the cilia were found to be static in half of the individuals, but completely or nearly normal in the others ${ }^{[10]}$. Variable diagnostic test results in nasal nitric oxide levels and ultrastructural images were also reported in patients with p.His154Pro mutation. Incomplete knowledge of phenotype could easily lead to underdiagnosis or false negatives. A larger number of patient subjects and detailed information was clearly needed to facilitate a better understanding of the subtle genotypephenotype correlations in PCD.

First reported in $2002^{[11]}, D N A H 5$ is the most frequent disease-causing gene in PCD patients. DNAH5 is an axonemal heavy chain dynein protein that functions as a force-generating protein to produce cilia bending. It has been reported that pathogenic variants in the $D N A H 5$ gene are responsible for nearly $15 \%$ to $29 \%$ of all PCD cases ${ }^{[12]}$. Several previous studies focused on patients with DNAH5, revealing that mutations in DNAH5 lead to absent or shorter dynein arms in respiratory cilia that are also mostly immotile or only show flickering movements. Patients usually have the typical clinical phenotype of PCD. In this study, we systematically evaluated the phenotype and genetic characteristics of 30 PCD patients carrying DNAH5 mutations. Intriguingly, mild respiratory phenotype, normal nasal nitric oxide and diverse ciliary beat pattern were identified in our cohort group. For specified patients, IF was performed to investigate outer dynein arm (ODA) perturbations in nasal ciliated cells. It is hoped that the detailed phenotype information identified in our study will help to better delineate the genotype-phenotype relationship of DNAH5.

\section{Materials And Methods}

\section{Patients and Clinical Materials}

The project was approved by the ethics committee of the Ethics board of The Children's Hospital of Fudan University, and written informed consent was obtained from all family members participating in this study. Inclusion criteria were as follows: 1) at least 2 out of 4 key clinical features (laterality defects, unexplained neonatal respiratory distress, year-round daily cough, or nasal congestion) or siblings of confirmed patients; and 2) HSVA suggestive of PCD and/or low nNO level. nNO and HSVA were measured repeatedly in cases with equivocal results. Exclusion criteria were confirmed diagnosis other than PCD (e.g., immune deficiency or cystic fibrosis). Eventually, a total of 114 patients belonging to 104 unrelated nuclear families with symptoms suggestive of PCD and positive screening test (nNO and/or HSVA) results were enrolled between January 1, 2016 and June 1, 2021 at the Children's Hospital of Fudan University. The clinical data, including lung function, high resolution CT (HRCT), and bacterial cultures were performed at enrollment. The diagnosis of PCD was based on the clinical findings, nNO, HSVA, TEM, and genetic testing, in accordance with the guidelines of European Respiratory Society ${ }^{[1]}$.

\section{Nasal Nitric Oxide Measurements}


Measurements of nNO were performed with the use of an EcoMedics CLD88 chemiluminescence NO analyzer (Duernten, Switzerland); the aspiration-sampling rate of $330 \mathrm{~nL} / \mathrm{min}$ was verified before and after each subject tested. The measurement of $\mathrm{nNO}$ in cooperative children was performed by breath hold maneuver for at least 10 s to close their velum as described previously ${ }^{[13]}$. For children that were uncooperative (less than 5 years old), nasal sampling was performed for 60 seconds during tidal breathing. Results were reported in $\mathrm{nL} / \mathrm{min}$ with the following equation: $\mathrm{nNO}(\mathrm{nL} / \mathrm{min})=\mathrm{NO}(\mathrm{ppb}) \times$ sampling rate $(\mathrm{nL} / \mathrm{min})$.

\section{Electron Microscopy and High-Speed Video Microscopy Analysis}

Nasal tissue was collected using a Rhino-Probe (Arlington Scientific, Springville, UT). To avoid secondary ciliary dyskinesia, nasal samples were taken in patients who free from acute airway infection for at least 4 weeks. The tissue was suspended in L-15 medium (Invitrogen, CA) immediately for analysis using a Leica inverted microscope (Leica DMI300B, Solms, Germany) with a 63× oil objective under differential interference contrast optics. Cilia beats were recorded at $200 \mathrm{frames} / \mathrm{sec}$ at room temperature $\left(25^{\circ} \mathrm{C}\right)$, using a 680 PROSILICA GE camera (Allied Vision, PA). At least ten videos were derived from each sample. The digital recordings were evaluated by two experienced but blinded investigators.

The ciliary ultrastructure was analyzed on nasal tissue, fixed in $2.5 \%$ glutaraldehyde, as described previously ${ }^{[13]}$. For each specimen, at least 30 transverse ciliary sections of different cells were used to evaluate the internal axonemal structure. Ciliary abnormalities were defined as the presence of defects in $>50 \%$ of cilia.

\section{Whole Exome Sequencing (WES) and Copy Number Variation (CNV) Analyses}

Blood samples were obtained from proband and available family members. Genomic DNA was extracted using the Gene Blood DNA Rapid Extraction Kit (Qiagen, China). WES was performed by Gemple Biotech Co. Ltd (Shanghai, China). Whole exome libraries were constructed using KAPA platform and KAPA Hyper Prep kit (Roche KAPA, Switzerland). Exomes were captured using SeqCap EZ MedExome (Roche NimbleGen) and sequenced by a HiSeq X Ten instrument (Illumina, San Diego, CA). Raw data was evaluated using FASTQC (version 0.11.5), and the linker sequences were removed by Cutadapt (version 1.10). The software BWA (version 0.7.15) was used to align the reads to the human reference genome GRCh37/hg19(UCSC). For analyzing the phenotypeassociated mutation sites, the mutation data were annotated and categorized according to the American College of Medical Genetics and Genomics (ACMG) guidelines ${ }^{[14]}$. Sanger sequencing was performed to validate the candidate variants, and segregation analyses were performed in family members. The panelcn.MOPS software was introduced to detect CNVs in WES data, which is a new algorithm that builds upon copy number estimation by Mixture of Poissons (cn.MOPS) to detect CNVs with high sensitivity and specificity ${ }^{[15]}$.

The RefSeq accession numbers of the transcript and corresponding protein isoform we used for mutation nomenclature were NM_001369.2 and NP_001360.1.

\section{Quantitative Real-Time Polymerase Chain Reaction (qPCR) Analysis}

To confirm CNVs detected by WES, qPCR was performed using DNA from patients, their parents and controls. DNA was obtained as described previously. qPCR was prepared using KAPA SYBR FAST qPCR Kit Master Mix(2X) Universal (Roche KAPA, Switzerland) on a CFX96 $6^{\text {TM }}$ Real-Time System instrument (BIORAD) and was performed on a LightCycler 480 System $\otimes$ (Roche diagnosistic) in triplicate. The reaction conditions were set at $95^{\circ} \mathrm{C}$ for 3 min, followed by 40 cycles of $95^{\circ} \mathrm{C}$ for $5 \mathrm{sec}$ and $55^{\circ} \mathrm{C}$ for $30 \mathrm{sec}$. The primer pair sequences were designed by NCBI (https://www.ncbi.nlm.nih.gov/). The comparative $\Delta \Delta \mathrm{Ct}$ method was used to calculate relative expression with data normalized to the mean level of an internal standard.

\section{Whole Genome Sequencing (WGS)}

Genomic DNA was obtained and sheared to fragments of an average length of 350 bp to construct libraries using KAPA platform and KAPA Hyper Prep kit (Roche KAPA, Switzerland). The libraries were then subjected to sequencing on the Illumina Hiseq X platform and 150 bp paired-end reads were generated. The same bioinformatics pipeline as described above for the exome sequencing analysis was used to analyze $D N A H 5$ mutations and precise breakpoints. The variants identified were confirmed by Sanger sequencing.

\section{IF Analysis}

Nasal tissue, obtained by nasal brushing biopsy, was suspended in cell culture medium and spread onto glass slides, then air dried. Samples were treated with $4 \%$ paraformaldehyde, $0.2 \%$ Triton-X 100 and $1 \%$ BSA before incubation with primary (overnight at $4{ }^{\circ} \mathrm{C}$ ) and secondary antibodies $(2 \mathrm{~h}$ at room temperature). Rabbit polyclonal anti-DNAH5 (HPA037470, Sigma, Sweden) and mouse monoclonal anti-alpha tubulin antibodies (ab24610, Abcam, UK) were used at a dilution of 1:1000. Highly cross-adsorbed secondary antibodies, goat anti-mouse Alexa Fluor 488 (1:1000) and goat anti-rabbit Alexa Fluor 647 (1:1000), were obtained from Abcam. Confocal images were taken using a Leica TCS SP8 confocal laser scanning microscope (Leica, Jena, Germany).

\section{Results}

\section{Clinical Phenotype of Individuals with DNAH5}

In the present study, we identified 30 Chinese patients with DNAH5 from 27 unrelated families. The age of affected individuals ranged from 0.6 to 43 years old and $60.0 \%$ (18/30) were males. PCD classical symptoms in our cohort included: Year-round wet cough (96.7\%, 29/30), neonatal respiratory distress (47.6\%, $10 / 21)$, nasosinusitis $(93.1 \%, 27 / 29)$, and chronic otitis media $(95.2 \%, 20 / 21)$. Further examination revealed that $80.0 \%$ of patients $(24 / 30)$ had situs inversus totalis and $7 \%$ of patients $(1 / 30)$ had heterotaxy; median forced expiratory volume in $1 \mathrm{~s}$ (FEV1) was estimated to be $74.6 \%$. In general, lung function, 
especially the forced expiratory flow at 25 and $75 \%$ of the pulmonary volume (FEF 25-75\%), gradually declined with age (Supplementary Figure 1). Surprisingly, as a 19-year-old boy, P40 had a mild clinical disease with normal lung function. Consistent with this, only small pieces of patchy exudation in his lower pulmonary lobes were identified by high resolution CT scans (Supplementary Figure 2). Patients P100 and P101 were from a Han Chinese consanguineous family (Figure 1A). No situs inversus totalis and bronchiectasis were identified in their mother (Figure 1B) and father (Figure 1C). While situs inversus totalis, bronchiectasis and nasosinusitis were found in P100 (Figure 1D-F), P101 (Figure 1G-I) and their older brother (not available). Surprisingly, the two older brothers had much earlier onset and more frequent lung infections than P100. Progressively lung failure leaded to P101 death shortly after diagnosis. Another met with lung failure and death at the age of 38 , according to their parents. HRCT indicated that P100 (Figure 1E) had a milder destruction of lung than P101 (Figure 1H) at enrollment.

$\mathrm{nNO}$ testing of 19 individuals revealed a median $\mathrm{nNO}$ production rate of $22.8 \mathrm{~nL} / \mathrm{min}$ (minimum, $5.6 \mathrm{~nL} / \mathrm{min}$; maximum, $99.0 \mathrm{~nL} / \mathrm{min}$ ). Interestingly, two siblings (P72 and P97) had nNO values that were above the disease-specific cut off value ( $77 \mathrm{~nL} / \mathrm{min})$. The detailed clinical characterizations and $\mathrm{nNO}$ values of our cohort are summarized in Table 1

\section{Ciliary Structural and Functional Analysis}

Ultrastructural analysis of 21 subjects with biallelic mutations in DNAH5 showed completely missing or shortened ODA. HSVA revealed that most DNAH5 mutant cilia were completely immotile or exhibited only occasional residual movement (Supplementary Video 1). However, four individuals (P24, P57, P69 and P97) with DNAH5 mutations exhibited diverse CBP. CBP of P24 was relatively stiff, with a decreased proximal bending (Supplementary Video 2). Cilia of P57 showed uncoordinated beating activity (Supplementary Video 3), while HSVA of P69 (Supplementary Video 4) and P97 (Supplementary Video 5) displayed a subtle reduction of the beating amplitude. As siblings, P72 and P97 carried the same mutations, but their ciliary beat patterns were distinctive. To further confirm the pathogenicity of the mutations, we studied P24, P57, P97 and P100 for DNAH5 localization in respiratory cells by IF, and found complete absence of DNAH5 in ciliary axonemes (Figure 2A-E). The details of ciliary structural and functional analysis are shown in Table 2.

\section{Genetic Characteristics and CDNA Analysis}

Collectively, 45 biallelic mutations were identified, of which 16 were missense variants, 5 were frameshift, 10 were nonsense variants, 2 were CNV, 2 were inframe deletions and 10 were splicing variants, while 37 variants (82.2\%) were novel (Table 3). Interestingly, the previously reported mutation c.5563dupA was found in four families. To find out whether the mutation was derived from a common ancestor (founder), we had sequenced 21 single-nucleotide polymorphisms (Supplementary Table 1) in the $200 \mathrm{~kb}$ DNAH5 locus to deduce the haplotypes. However, no identical haplotype was found in these families.

The WES results were routinely analyzed with an algorithm to detect CNVs. The positions of the CNV in the DNAH5 gene are shown in Figure $3 \mathrm{~A}$. A CNV causing heterozygous deletions corresponding to exons 71 to 72 of DNAH5 was suspected in P57 by analysis. Using qPCR, this deletion was recognized in P57 and her father (Figure 3B). As WES only covers exonic regions, WGS was performed to specify the precise breakpoints. Eventually, chr5:1371790913722659del was detected and confirmed (Figure 3D). Two CNVs causing homozygous deletions spanning exon 69-71 and exon 77-79 were suspected in P100 and P101; qPCR verified that exon 69-71 and exon 77-79 were homozygous deletions in P100, P101 and heterozygous deletions in their parents (Figure $3 C$ ). This indicated an autosomal recessive inheritance pattern conforming to the genetic disposition of PCD. To specify the precise breakpoints of the two CNVs, WGS was performed. Ultimately, chr5:13720087_13733030delinsGTTTTC and chr5:13649539_13707643del were detected and confirmed (Figure 3E).

\section{Discussion}

In the present study, the utility of NGS screening with additional CNV analysis was evaluated in a large cohort of 114 patients with highly suspected PCD from China. Overall, DNAH5 was the most prevalent mutant gene, and 45 different allelic mutations were identified in affected individuals from 27 ( $24.7 \%$ ) families. It suggested that the genetic profile in China was similar with those of the Europeans ${ }^{[16]}$. Multiple aspects of PCD characteristics, including clinical manifestations, ciliary phenotype, as well as genetic characterization were robustly obtained.

Several studies have systematically investigated associations between genotype and phenotype in PCD, which is a genetically heterogeneous disorder. It has been reported that lung function was significantly worse in individuals with CCDC39 and CCDC4O mutations and better in those with DNAH11 and DNAH5 mutations ${ }^{[17]}$. Consistent with the report of Shoemark et al, highly phenotypically diverse were also observed in our cohort ${ }^{[10]}$. For instance, $\mathrm{P} 40$ still retained nearly normal lung function in his adulthood with only a mild clinical phenotype. By contrast, P101 had severe clinical symptoms, and died of respiratory failure at middle age. Phenotypic diversity was in full display even among siblings. Despite frequent respiratory infections, P100 still had an apparently normal life at 38 years old with only mild to moderate impairment of pulmonary function. In contrast, his older brothers who carried identical mutations and were in the same living environment were not so fortunate; they all had severer bronchiectasis, and died from progressive lung failure at middle age. Similar heterogeneity was also observed in the pair of close-in-age sisters, P69 and P95. It is worth mentioning that data on mortality of PCD are extremely limited. To the best of our knowledge, the two respiratory deaths in our cohort are the first reported in DNAH5-associated PCD.

It has been widely reported that cilia beat patterns characterized in PCD patients correlate highly with the implicated gene ${ }^{[9]}$. The ODA generate the main mechanical force to produce cilia bending. It is generally recognized that mutations in genes encoding ODA and ODA docking components (e.g. DNAH5) cause absent or deficient ODA in cilia, leading to immotile cilia. Intriguingly, as reported here, we observed substantial heterogeneity of ciliary beat pattern in our patients. During HSVA, the CBP differed markedly in the range of static, restricted to stiff movement. The ciliary beat functions of $P 24$ (c.13126-2A $>G$, c.7789A>G), P57(c.13724-1G>A, 4.7kB del), P69(c.5563dupA, c.4355+5G>A) and P97(c.8311C>T, c.6646_6657del) were largely preserved. Similar phenomena were also observed by Blanchon et al, who identified $50 \%$ of cilia with normal motion in a patient carrying a DNAH5 missense mutation (p.R2771C) ${ }^{[18]}$. It appears that the different mutations have diverse effects on the protein, and that the alleles with severe effects on the ciliary beating parameters generated drastic structural and functional alterations in the protein. However, it is difficult to explain the difference of beat patterns observed in siblings. Similarly, 
variable ciliary beat pattern of PCD patients who carried identical mutation is also reported by Shoemark et al ${ }^{[10]}$. Further research is needed to better understand the mechanisms and influential factors of ciliary beat. It should be noted that HSVA provides important clues for the determination of pathogenicity of mutations. In recent years, genetic testing has rapidly advanced and become a viable diagnostic tool for PCD diagnosis ${ }^{[1,19]}$. In theory, a molecular genetic diagnosis of PCD can be established through the identification of biallelic pathogenic mutations in a known PCD-associated gene. In practice, however, frequent variants of unknown significance can make diagnosis equivocal, and in these situations, the concordance between genotype and phenotype becomes helpful. Overall, an insufficient knowledge of the ciliary phenotype for a specific genotype may lead to misdiagnosis.

CNVs have been shown to be associated with complex diseases such as cancer, neuropsychological disorders, Crohn disease, autism spectrum disorders, or multiple congenital anomalies ${ }^{[20]}$. As technology developed, roles of CNVs in the pathogenesis of PCD were gradually decoded. Deletions in SPAG1, ARMC4,

DYX1C1, LRRC50, ZYMD10 and DNAH5 were observed successively ${ }^{[21,22]}$, suggesting that CNV analysis should be used in the molecular evaluation of patients with PCD. Marshall reported that WES followed by targeted CNV analysis identification of 4 of 45 (8.8\%) PCD patients harbored clinically significant CNVs ${ }^{[22]}$. In a study by Fassad et al, CNVs accounted for $4 \%$ of variants overall in PCD patients ${ }^{[16]}$. In the present study, the CNVs were initially predicted by analysis with WES data plus panelcn.MOPS, a reliable algorithm to detect CNVs with high sensitivity and specificity, then confirmed by qPCR and Sanger sequencing. Through this process, we identified 2 of 27 (7.4\%) families with clinically significant CNVs. To further evaluate the pathogenicity, we performed high-resolution IF analyses, and confirmed that DNAH5 were absent in the cilia. A considerable proportion of CNV in PCD suggests that routine CNV analysis in the molecular diagnosis of PCD is clinically beneficial.

\section{Conclusions}

In conclusion, by utilizing state-of-the-art diagnostic techniques, we have provided a detailed description of the high diversity in DNAH5-associated PCD, which included clinical manifestations, ciliary phenotypes, and genetic spectrum. We hope that the diversity of phenotypes and genotypes identified by this study will lead to a better diagnosis of PCD and recognize the heterogeneous nature of this orphan disease.

\section{Abbreviations}

ACMG American College of Medical Genetics and Genomics

CNV Copy number variation

FEV1 Forced expiratory volume in the first second

HRCT High-resolution computed tomography

HSVA High-speed video analysis

IF Immunofluorescence

ODA Outer dynein arms

PCD Primary ciliary dyskinesia

qPCR Quantitative Real-Time Polymerase Chain Reaction

TEM Transmission electron microscopy

WES Whole Exome Sequencing

WGS Whole Genome Sequencing

\section{Declarations}

\section{Ethics approval and consent to participate}

The project was approved by the ethics committee of the Ethics board of The Children's Hospital of Fudan University, and written informed consent was obtained from all family members participating in this study.

\section{Consent for publication}

Parents or subjects gave informed written consent.

\section{Availability of data and materials}

The datasets used and/or analyzed during the current study are available from the corresponding author on reasonable request.

\section{Competing interests}

The authors declare that they have no competing interests. 


\section{Funding}

This work was supported by Shanghai Natural Science Foundation of Science and Technology Innovation Action Plan (Grant Number: 21ZR1409900 and 21ZR1410200).

\section{Authors' contributions}

ZYG and WCC performed the research, analyzed and interpreted the data, and drafted the manuscript. MRL performed experiments and prepared Figures 2 and Table 3. LBW and LLQ conceived and designed the study, revised the manuscript. The authors read and approved the manuscript to be published.

\section{Acknowledgements}

Not applicable.

\section{References}

[1] Lucas JS, Barbato A, Collins SA, et al. European Respiratory Society guidelines for the diagnosis of primary ciliary dyskinesia. Eur Respir J, 2017,49:1601090.

[2] Wallmeier J, Nielsen KG, Kuehni CE, et al. Motile ciliopathies. Nat Rev Dis Primers. 2020;6:77.

[3] Lucas JS, Davis SD, Omran H, et al. Primary ciliary dyskinesia in the genomics age. Lancet Respir Med. 2020;8:202-216.

[4] Pifferi M, Bush A, Mulé G, et al. Longitudinal Lung Volume Changes by Ultrastructure and Genotype in Primary Ciliary Dyskinesia. Ann Am Thorac Soc. 2021;18:963-970.

[5] Knowles MR, Ostrowski LE, Leigh MW, et al. Mutations in RSPH1 cause primary ciliary dyskinesia with a unique clinical and ciliary phenotype. Am J Respir Crit Care Med. 2014;189:707-17.

[6] Loges NT, Antony D, Maver A, et al. Recessive DNAH9 Loss-of-Function Mutations Cause Laterality Defects and Subtle Respiratory Ciliary-Beating Defects. Am J Hum Genet. 2018 Dec 6;103:995-1008.

[7] Antony D, Becker-Heck A, Zariwala MA, et al. Mutations in CCDC39 and CCDC40 are the major cause of primary ciliary dyskinesia with axonemal disorganization and absent inner dynein arms. Hum Mutat. 2013;34:462-72.

[8] Mitchison HM, Schmidts M, Loges NT, et al. Mutations in axonemal dynein assembly factor DNAAF3 cause primary ciliary dyskinesia. Nat Genet. 2012;44:381-9, S1-2.

[9] Raidt J, Wallmeier J, Hjeij R, et al. Ciliary beat pattern and frequency in genetic variants of primary ciliary dyskinesia. Eur Respir J. 2014;44:1579-88

[10] Shoemark A, Moya E, Hirst RA, et al. High prevalence of CCDC103 p.His154Pro mutation causing primary ciliary dyskinesia disrupts protein oligomerisation and is associated with normal diagnostic investigations. Thorax. 2018; 73:157-166.

[11] Olbrich H, Häffner K, Kispert A, et al. Mutations in DNAH5 cause primary ciliary dyskinesia and randomization of left-right asymmetry. Nat Genet. 2002;30:143-4.

[12] Zariwala MA, Knowles, MR, Leigh, MW. (2015). Primary ciliary dyskinesia. Retrieved from https://www.ncbi.nlm.nih.gov/books/NBK1122/

[13] Guo Z, Chen W, Wang L, et al. Clinical and Genetic Spectrum of Children with Primary Ciliary Dyskinesia in China. J Pediatr. 2020;225:157-165

[14] Richards S, Aziz N, Bale S, et al; ACMG Laboratory Quality Assurance Committee. Standards and guidelines for the interpretation of sequence variants: a joint consensus recommendation of the American College of Medical Genetics and Genomics and the Association for Molecular Pathology. Genet Med. 2015;17:405-24

[15] Povysil G, Tzika A, Vogt J, et al. panelcn.MOPS: Copy-number detection in targeted NGS panel data for clinical diagnostics. Human Mutation. 2017,38:889-897.

[16] Fassad MR, Patel MP, Shoemark A, et al. Clinical utility of NGS diagnosis and disease stratification in a multiethnic primary ciliary dyskinesia cohort. J Med Genet. 2020;57:322-330.

[17] Pifferi M, Bush A, Mulé G, et al. Longitudinal Lung Volume Changes by Ultrastructure and Genotype in Primary Ciliary Dyskinesia. Ann Am Thorac Soc. 2021;18:963-970

[18] Blanchon S, Legendre M, Bottier M, et al. Deep phenotyping, including quantitative ciliary beating parameters, and extensive genotyping in primary ciliary dyskinesia. J Med Genet. 2020;57:237-244

[19] Shapiro A J, Davis S D, Polineni D, et al. Diagnosis of Primary Ciliary Dyskinesia. An Official American Thoracic Society Clinical Practice Guideline. American Journal of Respiratory and Critical Care Medicine. 2018;197:e24-e39. 
[20] Harel T, Lupski JR. Genomic disorders 20 years on-mechanisms for clinical manifestations. Clin Genet. 2018;93:439-449.

[21] Takeuchi K, Xu Y, Kitano M, Chiyonobu K, Abo M, Ikegami K, et al. Copy number variation in DRC1 is the major cause of primary ciliary dyskinesia in the Japanese population. Mol Genet Genomic Med. 2020;8:e1137.

[22] Marshall CR, Scherer SW, Zariwala MA, Lau L, Paton TA, Stockley T, et al. Whole-Exome Sequencing and Targeted Copy Number Analysis in Primary Ciliary Dyskinesia. G3 (Bethesda). 2015;5:1775-81.

\section{Tables}

Table 1. Detailed clinical characteristics of the PCD patients with DNAH5 


\begin{tabular}{|c|c|c|c|c|c|c|c|c|c|c|c|c|c|}
\hline Case & Sex & Age at & Age at & $\mathrm{nNO}$ & RDS & Chronic & Sputum & $\begin{array}{l}\text { Pulm } \\
\text { pred }\end{array}$ & hary fun & tion \% & HRCT & nasosinusitis & otitis \\
\hline No & & Enrollment & Diagnosis & & & cough & pathogens & $\mathrm{FEV}_{1}$ & FVC & $\begin{array}{l}\text { FEF25- } \\
75\end{array}$ & & & media \\
\hline P5 & $\mathrm{F}$ & 10 & 10 & 10.4 & No & Yes & No & 82.9 & 61.6 & 27.1 & $\begin{array}{l}\text { Diffuse } \\
\text { nodules; Lobar } \\
\text { atelectasis; } \\
\text { Bronchiectasis }\end{array}$ & Yes & NA \\
\hline P9 & $\mathrm{F}$ & 16 & 14.2 & 10.3 & No & Yes & No & 87.6 & 75.0 & 41.0 & $\begin{array}{l}\text { Diffuse } \\
\text { nodules; Lobar } \\
\text { atelectasis; } \\
\text { Bronchiectasis }\end{array}$ & Yes & Yes \\
\hline P11 & $M$ & 1 & 1 & 9.1 & Yes & Yes & NA & NA & NA & NA & $\begin{array}{l}\text { Patchy } \\
\text { exudation }\end{array}$ & Yes & Yes \\
\hline P12 & M & 6 & 6 & 12.2 & Yes & Yes & $\mathrm{MC}$ & 84.7 & 80.1 & 41.6 & $\begin{array}{l}\text { Lobar } \\
\text { atelectasis }\end{array}$ & Yes & Yes \\
\hline P14 & $\mathrm{F}$ & 7 & 7 & 6.6 & Yes & Yes & No & 94.3 & 105.4 & 63.7 & $\begin{array}{l}\text { Lobar } \\
\text { atelectasis; }\end{array}$ & Yes & Yes \\
\hline $\mathrm{P} 21$ & M & 17 & 17 & 5.6 & NA & Yes & NA & 85.2 & 70.6 & 34.0 & NA & Yes & NA \\
\hline P24 & M & 5 & 5 & 29.5 & No & No & $\mathrm{HI}$ & NA & NA & NA & $\begin{array}{l}\text { Patchy } \\
\text { exudation }\end{array}$ & Yes & Yes \\
\hline P31 & $\mathrm{F}$ & 10.9 & 10.4 & 12.3 & Yes & Yes & NA & 96.0 & 65.0 & 41.2 & $\begin{array}{l}\text { Diffuse } \\
\text { nodules }\end{array}$ & Yes & Yes \\
\hline P35 & $M$ & 17 & 17 & NA & No & Yes & NA & 83.4 & 106.6 & 53.0 & NA & Yes & NA \\
\hline P36 & $M$ & 0.9 & 0.9 & NA & Yes & Yes & NA & NA & NA & NA & $\begin{array}{l}\text { Patchy } \\
\text { exudation; } \\
\text { Lobar } \\
\text { atelectasis }\end{array}$ & NA & NA \\
\hline P40 & $M$ & 17 & 17 & 13.5 & Yes & Yes & No & 97.2 & 109.8 & 89.9 & $\begin{array}{l}\text { Patchy } \\
\text { exudation; } \\
\text { Lobar } \\
\text { atelectasis }\end{array}$ & Yes & Yes \\
\hline P42 & $M$ & 13.7 & 11.7 & 14.7 & No & Yes & No & 86.0 & 60.7 & 33.8 & $\begin{array}{l}\text { Diffuse } \\
\text { nodules; }\end{array}$ & Yes & Yes \\
\hline P49 & $M$ & 10.3 & 10.3 & 13.2 & No & Yes & NA & 81.4 & 77.5 & 53.2 & $\begin{array}{l}\text { Diffuse } \\
\text { nodules; } \\
\text { Lobar } \\
\text { atelectasis; } \\
\text { Bronchiectasis; }\end{array}$ & Yes & Yes \\
\hline P57 & $\mathrm{F}$ & 0.6 & 0.6 & 8.1 & Yes & Yes & NA & NA & NA & NA & NA & Yes & No \\
\hline P58 & $M$ & 8 & 8 & 17.0 & No & Yes & $\mathrm{HI}$ & 81.8 & 83 & 59.7 & $\begin{array}{l}\text { Lobar } \\
\text { atelectasis; }\end{array}$ & Yes & Yes \\
\hline P69 & $\mathrm{F}$ & 28 & 28 & 42.9 & NA & Yes & Mab & 54.8 & 76.6 & 67.5 & $\begin{array}{l}\text { Lobar } \\
\text { atelectasis; } \\
\text { Bronchiectasis; }\end{array}$ & Yes & NA \\
\hline P70 & $M$ & 7 & 7 & 6.6 & No & Yes & No & 71.6 & 76.3 & 45.0 & $\begin{array}{l}\text { Diffuse } \\
\text { nodules ; } \\
\text { Lobar } \\
\text { atelectasis; } \\
\text { Bronchiectasis; }\end{array}$ & Yes & Yes \\
\hline P72 & $M$ & 9.8 & 9.8 & 99 & No & Yes & $\mathrm{HI}$ & 65.2 & 63.3 & 37.3 & $\begin{array}{l}\text { Diffuse } \\
\text { nodules; } \\
\text { Lobar } \\
\text { atelectasis; } \\
\text { Bronchiectasis; }\end{array}$ & No & Yes \\
\hline P77 & $\mathrm{F}$ & 38 & 38 & NA & NA & Yes & $\mathrm{KP}$ & 50.5 & 70.5 & 20.4 & NA & Yes & NA \\
\hline P78 & $M$ & 25 & 22 & NA & NA & Yes & NA & 35.8 & 73.6 & 18.9 & $\begin{array}{l}\text { Diffuse } \\
\text { nodules; } \\
\text { Lobar } \\
\text { atelectasis; } \\
\text { Bronchiectasis; }\end{array}$ & Yes & Yes \\
\hline P79 & $\mathrm{F}$ & 1.7 & 1.7 & 25.1 & Yes & Yes & No & NA & NA & NA & Normal & Yes & NA \\
\hline P92 & M & 29 & 29 & NA & NA & Yes & No & 81.4 & 112.0 & 34.0 & $\begin{array}{l}\text { Lobar } \\
\text { atelectasis; } \\
\text { Bronchiectasis; }\end{array}$ & Yes & Yes \\
\hline
\end{tabular}




\begin{tabular}{|c|c|c|c|c|c|c|c|c|c|c|c|c|c|}
\hline P94 & $\mathrm{F}$ & 4 & 4 & NA & No & Yes & NA & NA & NA & NA & $\begin{array}{l}\text { Patchy } \\
\text { exudation }\end{array}$ & Yes & Yes \\
\hline P95 & $\mathrm{F}$ & 29 & 29 & NA & NA & Yes & $\mathrm{HI}$ & 36.2 & 74.2 & 6.3 & NA & Yes & NA \\
\hline P96 & M & 24 & 24 & 14.8 & NA & Yes & NA & NA & NA & NA & NA & Yes & NA \\
\hline P97 & $\mathrm{F}$ & 6.0 & 6.0 & 82.5 & Yes & Yes & $\mathrm{HI}, \mathrm{MC}$ & 72.9 & 81.7 & 43.0 & $\begin{array}{l}\text { Lobar } \\
\text { atelectasis; } \\
\text { Bronchiectasis; }\end{array}$ & No & Yes \\
\hline P100 & $M$ & 38 & 38 & 9.9 & NA & Yes & NA & 62.2 & 62.7 & 27.0 & $\begin{array}{l}\text { Lobar } \\
\text { atelectasis; } \\
\text { Bronchiectasis; }\end{array}$ & Yes & Yes \\
\hline P101 & $M$ & 43 & 43 & NA & NA & Yes & NA & NA & NA & NA & $\begin{array}{l}\text { Lobar } \\
\text { atelectasis; } \\
\text { Bronchiectasis; }\end{array}$ & Yes & Yes \\
\hline P102 & $M$ & 2.5 & 2.5 & 3.6 & No & Yes & SA & NA & NA & NA & Normal & Yes & Yes \\
\hline P112 & $\mathrm{F}$ & 35 & 26 & NA & Yes & Yes & NA & NA & NA & NA & Bronchiectasis; & Yes & Yes \\
\hline
\end{tabular}

$A B$, acinetobacter baumannii; $A S D$, atrial septal defect; $C A$, central apparatus; $D C R V$, double chambered right ventricle; $F$, female; $F E F$ $25-75 \%$, forced expiratory flow at 25 and $75 \%$ of the pulmonary volume; $\mathrm{FEV}_{1}$, forced expiratory volume in the first second; FVC, forced vital capacity; HI, haemophilus influenza; HRCT, high-resolution computed tomography; HTX, heterotaxy; IDA, inner dynein arms; KP, Kleber pneumoniae; LP, legionella pneumophila; M, male; Mab, Mycobacterium abscessus; MC, moraxella catarrhalis; MTD: microtubular disarrangement; NA, not available; ODA: outer dynein arms; PA, seudomonas aeruginosa; RDS, respiratory distress; SA, staphylococcus aureus; SIT, situs inversus totalis; SS, situs solitus; VSD, ventricular septal defect.

Table 2. Detailed ciliary feature in patients with DNAH5 


\begin{tabular}{|c|c|c|c|}
\hline Case & TEM & CBP & $\mathrm{CBF}(\mathrm{Hz})$ \\
\hline P05 & Inconclusive & Minimal residual movements & 1.4 \\
\hline P09 & ODA/IDA & Immotile & 0 \\
\hline P11 & ODA/IDA & Minimal residual movements & 0 \\
\hline P12 & ODA & Immotile & 0 \\
\hline P14 & ODA & Minimal residual movements & 0 \\
\hline P21 & ODA & Immotile & 0 \\
\hline P24 & ODA & Restricted & 5.1 \\
\hline P31 & Inconclusive & Immotile & 0 \\
\hline P35 & ODA & NA & NA \\
\hline P36 & ODA & NA & NA \\
\hline P40 & ODA & Immotile & 0 \\
\hline P42 & ODA & Immotile & 0 \\
\hline P49 & Inconclusive & Minimal residual movements & 0 \\
\hline P57 & Inconclusive & Restricted & 2.1 \\
\hline P58 & ODA & Minimal residual movements & 0 \\
\hline P69 & NA & Restricted & 2.2 \\
\hline P70 & ODA & Minimal residual movements & 0 \\
\hline P72 & Inconclusive & Minimal residual movements & 0 \\
\hline P77 & NA & NA & NA \\
\hline P78 & NA & NA & NA \\
\hline P79 & NA & Minimal residual movements & 0 \\
\hline P92 & NA & NA & NA \\
\hline P94 & NA & NA & NA \\
\hline P95 & NA & NA & NA \\
\hline P96 & NA & NA & NA \\
\hline P97 & Inconclusive & Restricted & 4.0 \\
\hline P100 & ODA & Minimal residual movements & NA \\
\hline P101 & ODA & Immotile & 0 \\
\hline P102 & ODA & Minimal residual movements & 0 \\
\hline P112 & NA & NA & NA \\
\hline
\end{tabular}

IDA, inner dynein arms; NA, not available; ODA, outer dynein arms;

Table 3 DNAH5 mutations in primary ciliary dyskinesia 


\begin{tabular}{|c|c|c|c|c|c|c|c|c|c|}
\hline \multirow[t]{2}{*}{ Patient } & \multirow[t]{2}{*}{ Location } & \multirow[t]{2}{*}{ cDNA position } & \multirow[t]{2}{*}{ Zygosity } & \multirow{2}{*}{$\begin{array}{l}\text { Type of } \\
\text { variants }\end{array}$} & \multirow[t]{2}{*}{ Effect on protein } & \multirow[t]{2}{*}{ Ref(PMID } & \multirow[t]{2}{*}{ GnomAD, All } & \multicolumn{2}{|c|}{ ACMG Guideline } \\
\hline & & & & & & & & Class & Evidence \\
\hline \multirow[t]{2}{*}{ P05 } & Exon4 & $\begin{array}{l}\text { c.278- } \\
\text { 1_295delinsACAACAACAA }\end{array}$ & Comp. & Splice-site & / & / & None & $\mathrm{P}$ & PVS1;PME \\
\hline & Exon38 & c. $6304 C>T$ & Het & Missense & p.R2102C & / & 0.00002439 & LP & PM1;PM2. \\
\hline \multirow[t]{2}{*}{ P09 } & Exon54 & c.7579_7590del & Comp. & Inframe & p.2527_2530del & / & None & LP & PM2_Sup! \\
\hline & Exon32 & c. $5146 \mathrm{C}>\mathrm{T}$ & Het & Missense & p.R1716W & 19357118 & 0.000008125 & LP & PM1;PM2. \\
\hline \multirow[t]{2}{*}{ P11 } & Exon40 & c.6647delA & Comp. & Frameshift & p.K2216Rfs*20 & / & None & $\mathrm{P}$ & PVS1;PML \\
\hline & Exon45 & c. $7523 \mathrm{~T}>\mathrm{C}$ & Het & Missense & p.L2508P & & None & VUS & PM2_Sup| \\
\hline \multirow[t]{2}{*}{ P12 } & Exon37 & c. $6139 \mathrm{C}>\mathrm{T}$ & Comp. & Nonsense & p.Gln2047* & / & None & $\mathrm{P}$ & PVS1;PM2 \\
\hline & Exon 14 & c. $1852 C>T$ & Het & Nonsense & p.Arg618* & 29363216 & 0.000004102 & $\mathrm{P}$ & PVS1; PM: \\
\hline \multirow[t]{2}{*}{ P14 } & Exon45 & c. $7469 \mathrm{G}>\mathrm{A}$ & Comp. & Nonsense & p.W2490* & / & None & $\mathrm{P}$ & PVS1; PM: \\
\hline & Exon55 & c. $9235 \mathrm{C}>\mathrm{T}$ & Het & Nonsense & p.R3079* & / & None & $\mathrm{P}$ & PVS1; PM: \\
\hline P21 & Exon27 & c. $4193 A>G$ & Hom & Missense & p.Q1398R & / & None & VUS & PM2_Sup| \\
\hline \multirow[t]{2}{*}{ P24 } & Intron75 & c. $13126-2 A>G$ & Comp. & Splice-site & / & / & None & LP & PVS1_Mor \\
\hline & Exon47 & c. $7789 A>G$ & Het & Missense & p.T2597A & / & None & LP & PM1;PM2; \\
\hline \multirow[t]{2}{*}{ P31 } & Exon34 & c.5563dupA & Comp. & Frameshift & p. $11855 \mathrm{Nfs}^{\star} 6$ & 31118369 & 0.00006099 & $P$ & PVS1;PME \\
\hline & Intron79 & c. $13724-1 \mathrm{G}>\mathrm{C}$ & Het & Splice-site & / & / & None & LP & PVS1_MoI \\
\hline \multirow[t]{2}{*}{ P35 } & Exon60 & c. $9897+1 G>A$ & Comp. & Splice-site & l & / & None & VUS & PVS1_MoI \\
\hline & Exon50 & c. $8265 C>G$ & Het & Missense & p.F2755L & / & None & VuS & PM1;PM2. \\
\hline \multirow[t]{2}{*}{ P36 } & Exon49 & c. $8030 \mathrm{G}>\mathrm{A}$ & Comp. & Missense & p.R2677Q & 24150548 & 0.00002035 & LP & PM1;PM2. \\
\hline & Exon32 & c. $5150 \mathrm{~T}>\mathrm{A}$ & Het & Missense & p.F1717Y & / & None & LP & PM1;PM2. \\
\hline \multirow[t]{2}{*}{ P40 } & Exon51 & c. $8498 \mathrm{G}>\mathrm{A}$ & Comp. & Missense & p.R2833H & 27637300 & 0.0000122 & LP & PM1;PM2. \\
\hline & Exon 12 & c.1616_1617delAG & Het & Frameshift & p.E539Vfs*6 & / & None & $\mathrm{P}$ & PVS1;PML \\
\hline \multirow[t]{2}{*}{ P42 } & Exon34 & c.5563dupA & Comp. & Frameshift & p. $.11855 N^{\prime} s^{\star} 6$ & 31118369 & 0.00006099 & $\mathrm{P}$ & PVS1;PME \\
\hline & Exon27 & c.4147_4148delAinsTCC & Het & Frameshift & p.I1383Sfs*22 & / & None & $\mathrm{P}$ & PVS1;PML \\
\hline \multirow[t]{2}{*}{ P49 } & Exon56 & c. $9502 \mathrm{C}>\mathrm{T}$ & Comp. & Nonsense & p.R3168* & / & 0.0000122 & $\mathrm{P}$ & PVS1;PMé \\
\hline & Exon48 & c. $7912 \mathrm{~A}>\mathrm{T}$ & Het & Nonsense & p.K2638* & / & None & $\mathrm{P}$ & PVS1;PM2 \\
\hline \multirow[t]{2}{*}{ P57 } & Intron79 & c. $13724-1 \mathrm{G}>\mathrm{A}$ & Comp. & Splice-site & / & l & None & LP & PVS1_Mor \\
\hline & Exon $71-72$ & $4.7 \mathrm{kB}$ del & Het & CNV & / & / & None & LP & PVS1_Mor \\
\hline \multirow[t]{2}{*}{ P58 } & Intron53 & c. $8821-2 A>C$ & Comp. & Splice-site & I & / & None & LP & PVS1_MoI \\
\hline & Exon7 & c.877dupA & Het & Frameshift & p.R293fs & / & 0.000004065 & $\mathrm{P}$ & PVS1;PM2 \\
\hline P69 & Exon34 & c.5563dupA & Comp. & Frameshift & p. $11855 \mathrm{Nfs}^{\star} 6$ & 31118369 & 0.00006099 & $\mathrm{P}$ & PVS1;PME \\
\hline & Intron27 & c. $4355+5 G>A$ & Het & Splice-site & / & l & 0.0000449 & VUS & PM3;PP1;I \\
\hline P70 & Intron 10 & c. $1320+1 G>A$ & Comp. & Splice-site & / & / & None & LP & PVS1_MoI \\
\hline & Exon34 & c. $5557 \mathrm{~A}>\mathrm{T}$ & Het & Nonsense & p.K1853* & 25525159 & 0.000008133 & $\mathrm{P}$ & PVS1;PMé \\
\hline P72 & Exon50 & c. $8311 \mathrm{C}>\mathrm{T}$ & Comp. & Missense & p.R2771C & l & 0.00003658 & $\mathrm{P}$ & PM1;PM2. \\
\hline & Exon40 & c.6646_6657del & Het & inframe & p.K2216_Y2219del & l & None & $\mathrm{P}$ & PM2_Sup| \\
\hline P77 & Exon38 & c. $6305 \mathrm{G}>\mathrm{A}$ & Comp. & Missense & p.R2102H & / & 0.000004065 & VUS & PM1;PM2. \\
\hline & Exon19 & c. $2980 \mathrm{C}>\mathrm{T}$ & Het & Missense & p.R994W & I & 0.0002 & VUS & PP4 \\
\hline P78 & Exon79 & c. $13746 C>G$ & Comp. & Nonsense & p.Y4582* & / & None & VUS & PVS1_Mor \\
\hline & Exon48 & c. $7979 A>G$ & Het & Splice-site & / & / & None & VUS & PM1;PM2. \\
\hline P79 & Exon45 & c.7579_7590del & Comp. & inframe & p.T2527_D2530del & / & None & LP & PM2_Sup| \\
\hline & Exon31 & c. $5047 \mathrm{G}>\mathrm{T}$ & Het & Nonsense & p.E1683* & l & None & $\mathrm{P}$ & PVS1;PML \\
\hline
\end{tabular}




\begin{tabular}{|c|c|c|c|c|c|c|c|c|c|}
\hline P92 & Exon34 & c.5563dupA & Comp. & Frameshift & p. $11855 \mathrm{Nfs}^{\star} 6$ & 31118369 & 0.00006099 & $P$ & PVS1;PME \\
\hline & Exon63 & c. $10616 \mathrm{G}>\mathrm{A}$ & Het & Missense & p.R3539H & 22416021 & 0.00002038 & LP & PM1;PM2. \\
\hline \multirow[t]{2}{*}{ P94 } & Intron76 & c. $13339-1 \mathrm{G}>\mathrm{A}$ & Comp. & Splice-site & / & / & None & $P$ & PVS1;PM2 \\
\hline & Exon36 & c. $5971 A>G$ & Het & Missense & p.K1991E & / & None & LP & PM1;PM2. \\
\hline \multirow[t]{2}{*}{ P95 } & Exon34 & c.5563dupA & Comp. & Frameshift & p.I1855Nfs*6 & 31118369 & 0.00006099 & $P$ & PVS1;PME \\
\hline & Intron27 & c. $4355+5 G>A$ & Het & Splice-site & / & / & 0.0000449 & VUS & PM3;PP1;I \\
\hline \multirow[t]{2}{*}{ P96 } & Exon68 & c. $11664 C>G$ & Comp. & Nonsense & p.Y3888* & / & None & $P$ & PVS1;PMÉ \\
\hline & Exon34 & c.5563dupA & Het & Frameshift & p.I1855Nfs*6 & 31118369 & 0.00006099 & $P$ & PVS1;PME \\
\hline \multirow[t]{2}{*}{ P97 } & Exon50 & c. $8311 \mathrm{C}>\mathrm{T}$ & Comp. & Missense & p.R2771C & / & 0.00003658 & $P$ & PM1;PM2. \\
\hline & Exon40 & c.6646_6657del & Het & Nonframeshift & p.K2216_Y2219del & / & None & $P$ & PM2_Supl \\
\hline P100 & $\begin{array}{l}\text { Exon69- } \\
71 \text { Exon77- } \\
79\end{array}$ & $58.1 \mathrm{kB}, 12.9 \mathrm{kB}$ del & Hom & CNV & / & / & None & LP & PVS1_Mor \\
\hline P101 & $\begin{array}{l}\text { Exon69- } \\
71 \text { Exon77- } \\
79\end{array}$ & $58.1 \mathrm{kB}, 12.9 \mathrm{kB}$ del & Hom & CNV & / & / & None & LP & PVS1_Mor \\
\hline \multirow[t]{2}{*}{ P102 } & Exon63 & c. $10616 \mathrm{G}>\mathrm{A}$ & Comp. & Missense & p.R3539H & 22416021 & 0.00002038 & LP & PM1;PM2. \\
\hline & Exon34 & c. $5648 \mathrm{G}>\mathrm{C}$ & Het & Missense & p.R1883P & / & None & VUS & PM2_Supl \\
\hline \multirow[t]{2}{*}{ P112 } & Intron69 & c. $11883+2 T>C$ & Comp. & Splice-site & I & / & None & $P$ & PVS1;PM 2 \\
\hline & Exon63 & c. $10616 \mathrm{G}>\mathrm{A}$ & Het & Missense & p.R3539H & 22416021 & 0.00002038 & LP & PM1;PM2. \\
\hline
\end{tabular}

PMID®PubMed Unique Identifier; HGMD, Human Gene Mutation Database; NA, not available;

\section{Figures}

A
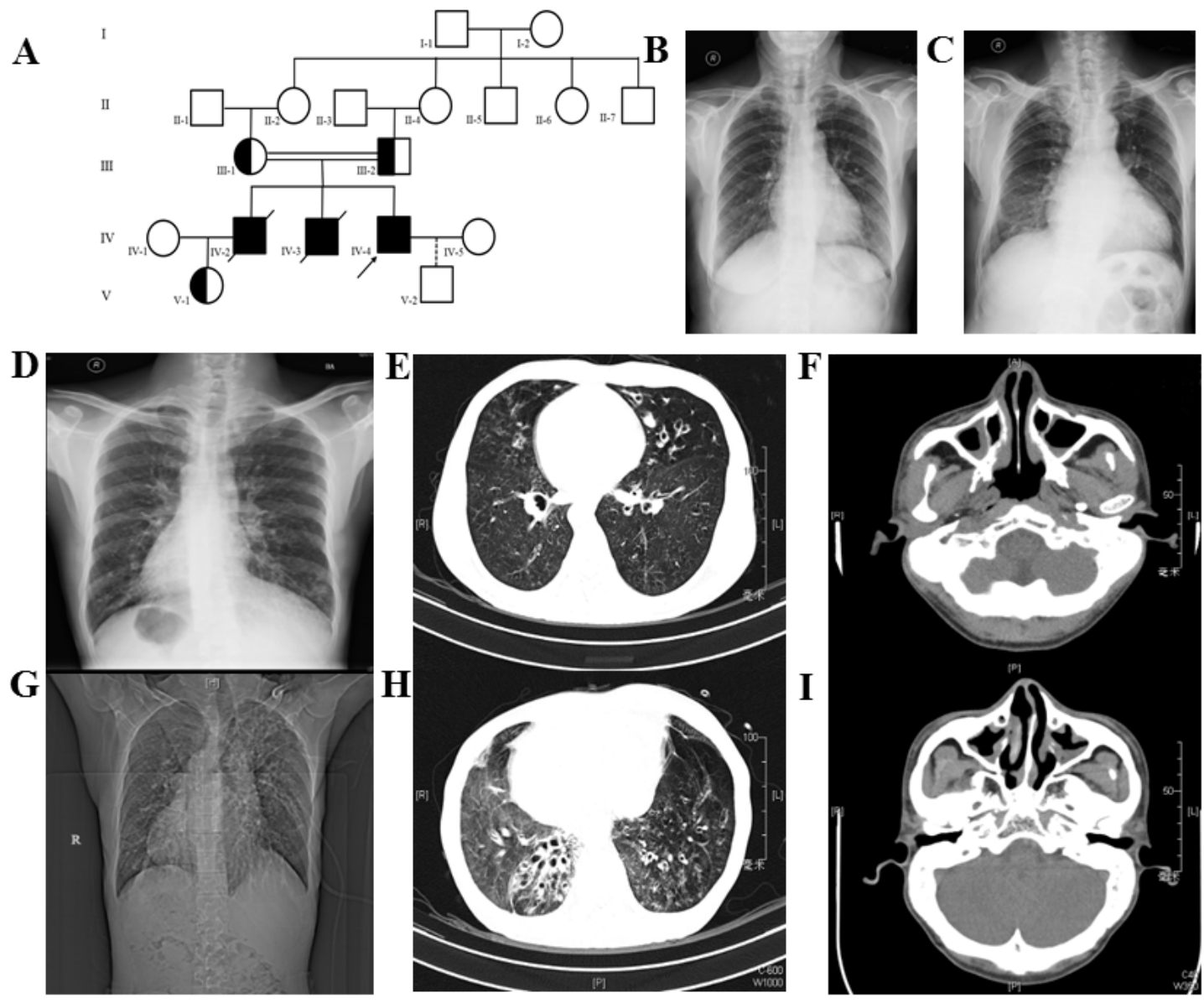


\section{Figure 1}

Pedigree structure and clinical examinations of P100's family

(A) Pedigree structure of P100's family. Arrow denotes the proband P100. Affected patients are presented by black symbols; half black symbol indicates heterozygous deletions of $D N A H 5$; symbols with diagonal slashes denote deceased individuals. CXR images of the mother (B) and father (C) of proband shows normal distribution of organs. Clinical imaging show mirrored distributed viscera (D) and features of bronchiectasis $(E)$ and paranasal sinusitis $(F)$ of P100. Clinical imaging of P101 show distinctive situs inversus (G) and severer bronchiectasis (H) and paranasal sinusitis (I), compared to P100.

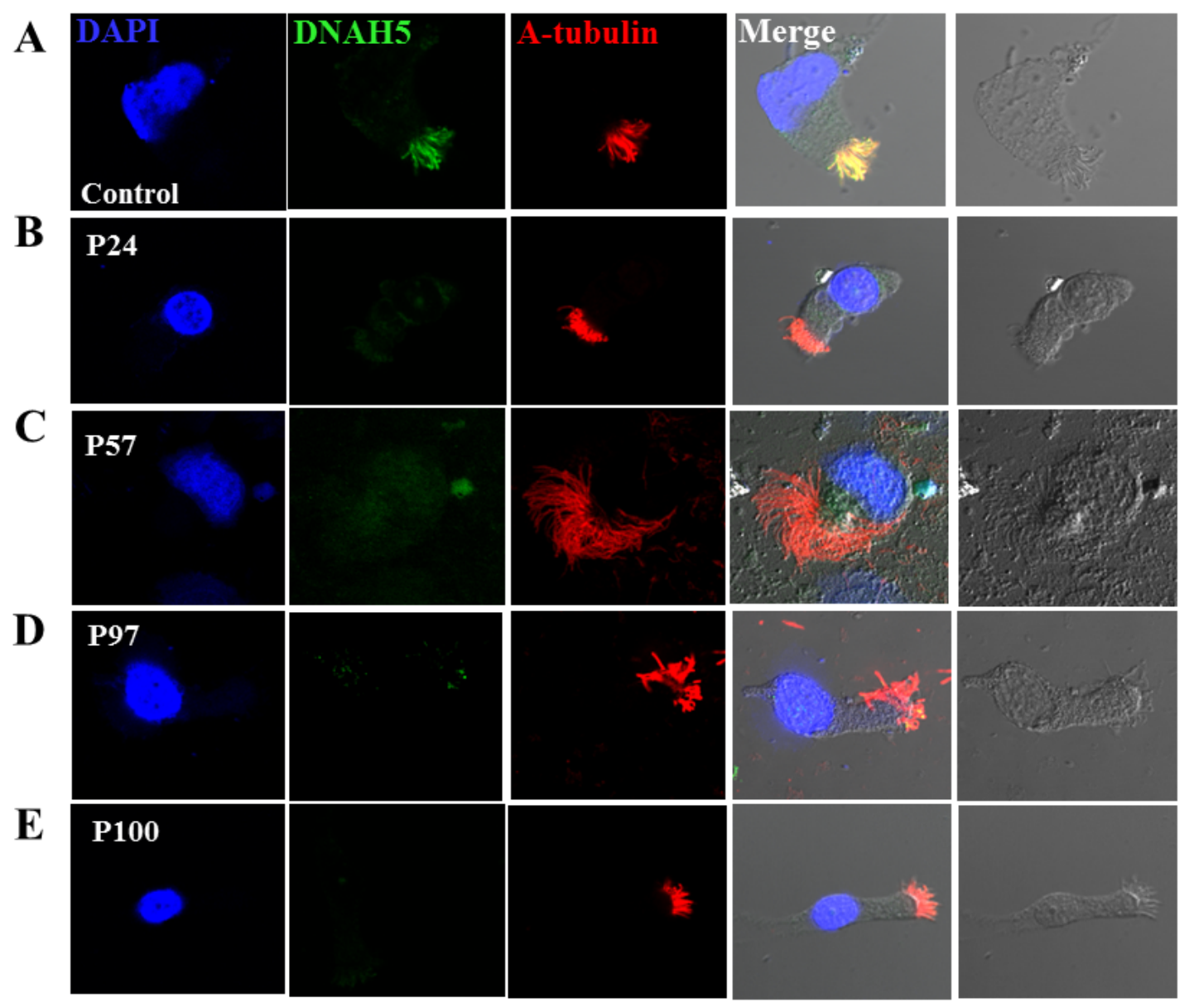

\section{Figure 2}

Subcellular localization of DNAH5 in respiratory epithelial cells.

Axoneme-specific antibodies against acetylated a-tubulin (red) were used as the control. In respiratory epithelial cells from healthy probands (A), DNAH5 (green) localized predominantly along the entire length of the axonemes, as well as to the apical cytoplasm. In respiratory epithelial cells from individuals P24 (B), P57 (C), P97 (D) and P100 (E), DNAH5 was absent from the axoneme and markedly reduced in the apical cytoplasm. 
A

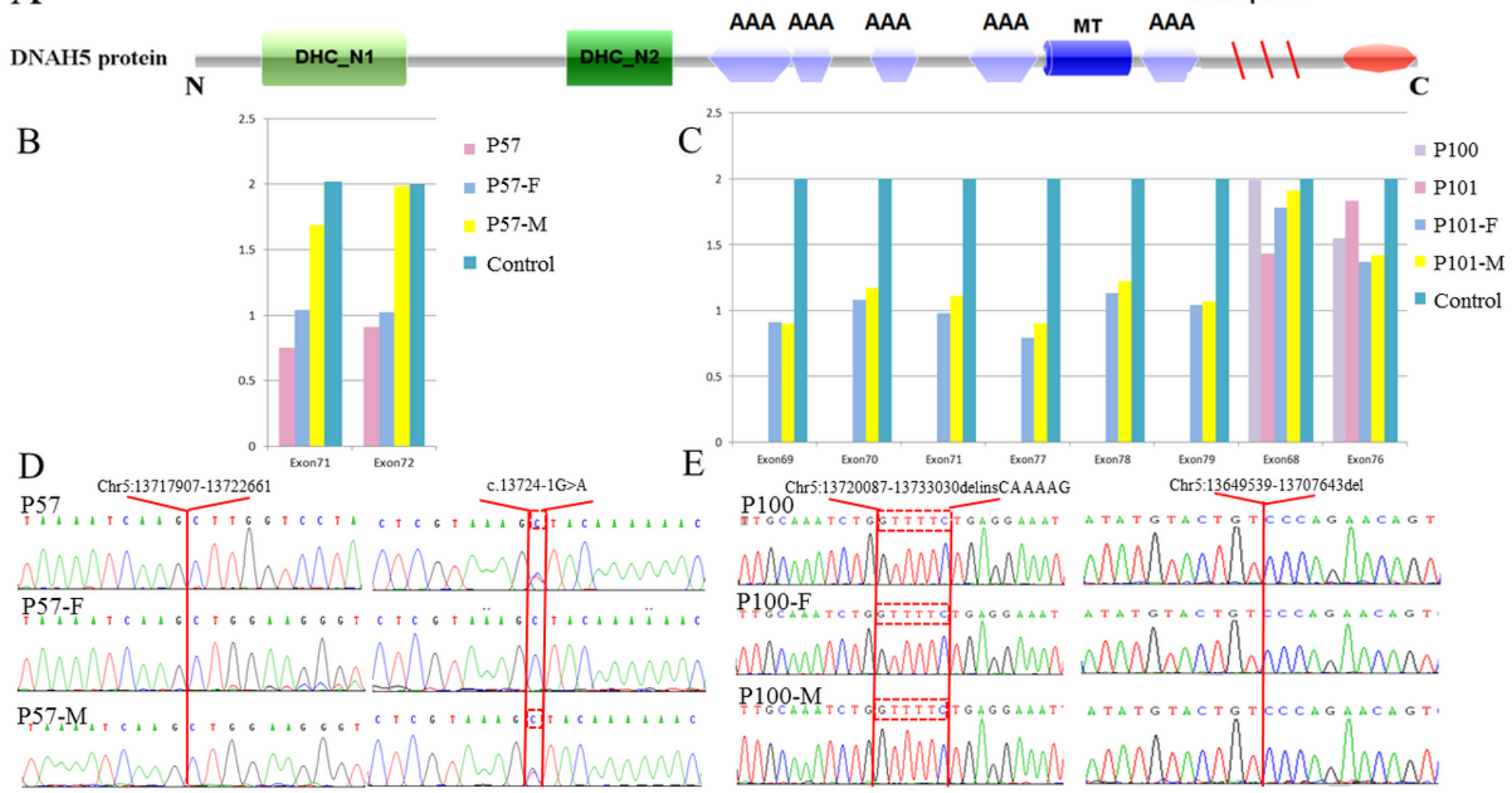

Figure 3

CNV analysis by qPCR and Sanger sequencing.

(A) Schematic of DNAH5 protein structure, the red slash indicating the position of the CNVs. qPCR analysis of relative DNA content in whole blood from the control subject, P57 (B), P100 (C) and their family member. Sanger sequencing confirmed the CNVs at Chr5:13717907-13722661 in P57 (D) and chr5:13720087_13733030, chr5:13649539_13707643 in P100 (E).

\section{Supplementary Files}

This is a list of supplementary files associated with this preprint. Click to download.

- SupplementaryFigure1.tif

- SupplementaryFigure2.tif

- Supplementarytable1.docx

- Supplementaryvideo1.mp4

- Supplementaryvideo2.mp4

- Supplementaryvideo3.mp4

- Supplementaryvideo3.mp4

- Supplementaryvideo4.mp4

- Supplementaryvideo5.mp4 